\title{
Diagnostic Accuracy of Imaging Techniques for Rotator Cuff Pathology: An Evidence-to-Practice Review
}

\author{
Hayley O'Connell, SCAT, ATC; Brittany deCamp, SCAT, ATC; and Zachary Winkelmann, PhD, SCAT, ATC
}

University of South Carolina, Columbia, SC

\begin{abstract}
When comparing imaging techniques for rotator cuff pathologies, clinicians tend to consider musculoskeletal ultrasonography (MSK US), magnetic renaissance imaging (MRI), and magnetic renaissance imaging with arthrogram (MRA) as diagnostic imaging techniques. Since the most recent systematic review on imaging conducted in 2003, imaging technology has improved, indicating the need for a metaanalysis to evaluate the accuracy of new diagnostic techniques (MSK US) for evaluating rotator cuff pathologies. The accuracy of MSK US readings between radiologists and non-radiologists was also analyzed. Data were extracted from three different databases and included articles exploring diagnostic imaging and the accuracy of technique at the shoulder joint. All research findings were then rated for any risk of bias using the revised version of the quality assessment of diagnostic accuracy studies (QUADAS-2). Statistically, the authors used hierarchical summary receiveroperating characteristics to compare accuracy of diagnostic imaging techniques across the literature. The results from the guiding manuscript indicated that MSK US, MRI, and MRA were considered highly sensitive diagnostic imaging techniques for full-thickness rotator cuff tears (Sn: US: 0.860.94, MRI: 0.85-0.95, MRA: 0.83-0.95). For partial-thickness rotator cuff tears, likelihood ratios indicated an increased accuracy in MRA diagnostic ability (Sn: MRA: 0.83 ), though MSK US and MRI were still considered highly sensitive for diagnosing partial-thickness rotator cuff tears (Sn: MSK US: 0.68$, MRI: 0.67$)$. When comparing radiologists and nonradiologists use of MSK US, there was no significant difference in diagnostic accuracy based on the reading provider. As the diagnosis based on imaging determines the need for surgical intervention, the guiding review indicated that all three diagnostic tools (MSK US, MRI and MRA) were considered highly sensitive for rotator cuff pathologies (average SN of all diagnostic tools: 0.90-0.91). Determining which imaging technique to use should be based on patientcentered factors, such as possibility of the presence of other shoulder pathology, invasiveness of the procedure, and financial implications. Diagnostic MSK US optimizes these factors, in addition to being highly sensitive.
\end{abstract}

\section{Key Phrases}

Diagnostic testing and physical examination: upper extremity; college and university patient population, clinic and hospital patient population

Copyright (C) by Indiana State University All rights reserved. ISSN Online 2577-8188

\section{Correspondence}

Dr. Zachary Winkelmann, University of South Carolina

1300 Wheat Street, Columbia, SC 29208.

E-mail:winkelz@mailbox.sc.edu

Twitter: @zachwinkelmann

\section{Full Citation}

O'Connell H, deCamp B, Winkelmann ZK. Diagnostic accuracy of imaging techniques for rotator cuff pathology: an evidence-to-practice review. Clin Pract Athl Train. 2020;3(3): 1 2-17.

https://doi.org/10.31622/2020/000.3.3.

Submitted: April 20, 2020 Accepted: August 17, 2020

\section{ORIGINAL REFERENCE}

Roy JS, Braen C, Leblond J, et al. Diagnostic accuracy of ultrasonography, MRI, and MR arthrography in the characterization of rotator cuff disorders: a systematic review and meta-analysis. $\mathrm{Br}$ J Sports Med. 2015;49(20):1316-1328

\section{SUMMARY}

\section{CLINICAL PROBLEM AND QUESTION}

\section{Shoulder pathologies affect much of the general}

population and most commonly are a result of injury to the rotator cuff tendons.' The rotator cuff is the group of muscles (supraspinatus, infraspinatus, teres minor and subscapularis) that are primarily responsible for external and internal rotation of the shoulder along with assisting in stabilizing the glenohumeral joint during a majority of all other shoulder movements. and in stabilizing the glenohumeral joint. Conditions of the rotator cuff often constitute rotator cuff tendinopathies, partial thickness rotator cuff tears, and full thickness rotator cuff tears. ${ }^{2}$ Without treatment, such as therapeutic rehabilitation or surgical intervention, patients Volume 3 - Issue 3 - November 2020 
suffering from a rotator cuff pathology may experience lifelong pain and significant functional limitations. ${ }^{2}$ Surgical intervention is often indicated and time-sensitive for individuals with full thickness rotator cuff tears, so an accurate diagnosis is critical. ${ }^{3}$ The three diagnostic imaging techniques tools to determine the degree of injury and guide treatment are musculoskeletal ultrasonography (MSK US), magnetic resonance imaging (MRI) and magnetic resonance arthrography (MRA). Musculoskeletal ultrasound (often referred to as diagnostic ultrasound) is the use of a sound wave through a transducer to visualize the structures below. The MRI and MRA are both operated by using a high-powered magnet within the machine to formulate an image to be interpreted by a radiologist. The sole difference between MRI and MRA is the joint being evaluated is injected with medical dye before an MRA scan to help define the structures of concern. ${ }^{1}$ Since each diagnostic imaging technique's last systematic review (20102015) of diagnostic accuracy, new research and advanced technology have emerged including a more widespread use $(+347 \%$ between 2003 2015) of musculoskeletal ultrasonography nonradiologists (radiology us decreased by $28 \%$ from 2003-2015).4,5 In sports medicine cases, a re-evaluation of the diagnostic imaging techniques is warranted. A new statistical measure, the hierarchical summary receiveroperating characteristic (HSROC), has been validated for diagnostic accuracy and guides the study recommendations for utilization of MSK US, MRI and MRAI. HSROC allows researchers to take into account within and between study variability and better differentiates different thresholds of study results. ${ }^{6-8}$ The purpose of the guiding systematic review study was to evaluate the diagnostic accuracy of MSK US, MRI, and MRA for rotator cuff pathologies. The secondary aim was to determine the accuracy of improved technology in diagnosing rotator cuff tears and to assess the use of MSK US when utilized by radiologists as compared to non-radiologists. Understanding the diagnostic accuracy of MSK US, MRI and MRA is important to better guide intervention strategies, especially in regard to making surgical recommendations, in addition to the benefits regarding patient-centered care (i.e. less cost, time, and a less invasive of the procedure).

\section{SUMMARY OF LITERATURE}

The guiding systematic review and meta-analysis authors used Medline, EMBASE and CINAHL in their search for articles published before 2014 . Articles were included if they 1) included adults participants with shoulder pain, 2) utilized MSK US, MRI, and/or MRA as a diagnostic tool and surgery as a reference standard, and 3) reported on the diagnostic accuracy of the imaging techniques in diagnosing a rotator cuff pathology. ${ }^{4}$ The search of the databases revealed 264 studies that met criteria for a full review. Studies were excluded due to incomplete data, small population size, and the use of a different index/diagnostic test. After study exclusion based on two evaluator analyses, 82 studies were included in the meta-analysis. Of these, 47 articles included MSK US studies, 29 articles were included for MRI, and 21 articles were included for MRA. These articles were then analyzed for bias using the Quality Assessment Tool for Diagnostic Accuracy Studies (QUADAS-2) assessing four main components of bias including patient selection bias, the diagnostic test utilized, the reference standard (surgery), and flow and timing in regard to patient retention and speed of intervention. ${ }^{9}$ Of the studies included, most had a high risk of bias in 3 of the 4 QUADAS-2 categories. ${ }^{9}$ As the reference standard is an invasive procedure (surgery), researchers noted that some bias could not be avoided, particularly in participant selection.

\section{SUMMARY OF OUTCOMES}

Data was extracted based on participant characteristics, index test, diagnostic accuracy (sensitivity and specificity) and based on the reference standard. The researchers of the 
guiding systematic review utilized the HSROC to evaluate the diagnostic accuracy of the three different diagnostic imaging techniques with a specific focus on test settings and technology. The HSROC is a statistical measure used to determine the overall sensitivity and specificity of diagnostic testing. ${ }^{6}$ This statistical measure was utilized as it considers between-study and within-study variability and ultimately provides a receiver operating curve, graphing specificity over sensitivity to indicate the tool's accuracy. ${ }^{9}$ In addition to analyzing diagnostic accuracy, this measure was also used to compare MRI magnet strength and MSK US transducer frequency levels to guide the best practice use of these tools. Accuracy of radiologist and non-radiologist use of the MSK US was also calculated. Results were deemed clinically important only if variation was beyond the error associated with the accuracy scores. This, along with the analysis of bias through the QUADAS-2 were combined and used to formulate diagnostic tool recommendations.

\section{FINDINGS AND CLINICAL IMPLICATIONS}

The guiding systematic review and meta-analysis suggests that there are no significant differences between the accuracy of the three diagnostic imaging techniques based on their specificity and sensitivity found using the HSROC statistics. ${ }^{8}$ Diagnostic imaging techniques were assessed for accuracy diagnosing rotator cuff tendinopathy (Figure 1), rotator cuff partial-thickness tears (Figure 2), and full-thickness rotator cuff tears (Figure 3). The HSROC indicated that MSK US had an overall sensitivity of $0.79195 \%$ confidence interval $[\mathrm{Cl}]=0.63-0.91$ ) and specificity of 0.94 $(95 \% \mathrm{Cl}=0.86-0.99)$. For partial-thickness tears, the literature indicated a sensitivity of 0.68 $(95 \% \mathrm{Cl}=0.54-0.83)$ and specificity of 0.94 $(95 \% \mathrm{Cl}=0.90-0.97)$ while full-thickness rotator cuff tears, sensitivity was $0.91(95 \% \mathrm{Cl}=0.86$ $0.94)$ and specificity was $0.93(95 \% \mathrm{Cl} 0.91$ 0.96). For most of these findings, the sensitivity and specificity for MSK US were considered high

Copyright $($ by Indiana State University All rights reserved. ISSN Online 2577-8188 enough to rule in and/or out suspected rotator cuff pathologies. In addition to assessing the accuracy of the diagnostic test, information was extracted that indicated the qualifications of radiologists or non-radiologist healthcare personnel in reading the MSK US, and their ability to accurately diagnose a rotator cuff pathology using this diagnostic tool. With the reference standard being surgical diagnosis of pathology for all conditions, they found no significant difference (Radiologist - Sn: 0.89, Sp: 0.85; Sonographers \& Orthopaedists - Sn: 0.88, Sp: 0.89) in accuracy of the reading provider. While this finding supports the use of MSK US without preference of the individual reading the images, the authors noted that all non-radiologists were specifically trained in the use of diagnostic ultrasound, which could explain the consistencies in imaging interpretations, even when considering alterations in MSK US transducer frequency. Transducer frequency in diagnostic MSK US on average ranges from $5 \mathrm{MHz}$ to $20 \mathrm{MHz}$, with the most widely accepted use being set for $7.5 \mathrm{MHz}$. The guiding manuscript indicated that there are no improvements in image quality at transducer frequencies above or below the generally accepted $7.5 \mathrm{MHz} .^{8}$

The findings also indicated that MRI is a highly specific tool. For partial-thickness rotator cuff tears, MRI has a sensitivity of $0.67195 \% \mathrm{Cl}=$ $0.50-0.82)$ and a specificity of $0.94(95 \% \mathrm{Cl}$ 0.88 - 0.99). For full-thickness tears in the rotator cuff, MRI was found to have an overall sensitivity of $0.90(95 \% \mathrm{Cl}=0.85-0.95)$ and specificity of $0.93(95 \% \mathrm{Cl}=0.89-0.97)$. There was minimal variation in the sensitivity and specificity numbers between MRI and MSK US. For MRI, Volume 3 - Issue 3 - November 2020 
Figure 1. Full-thickness or partial thickness RC tears

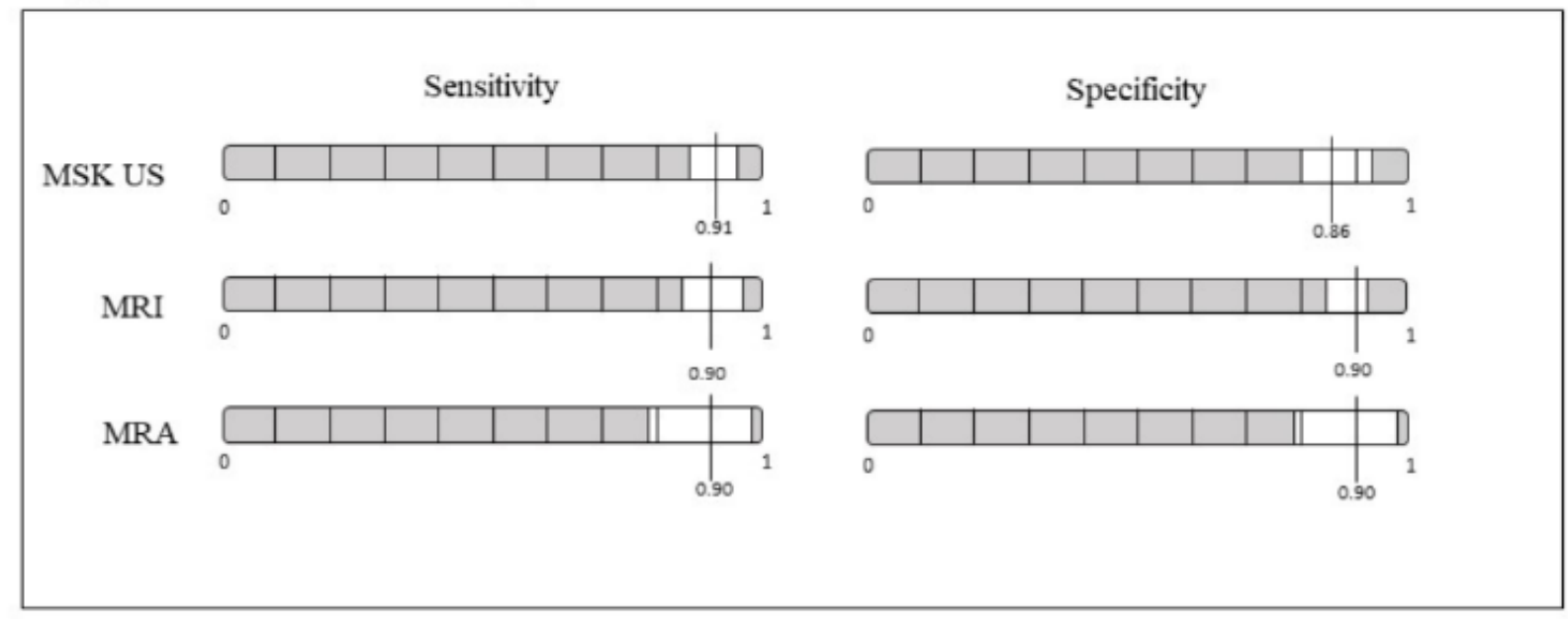

Figure 2 Full-thickness RC tears

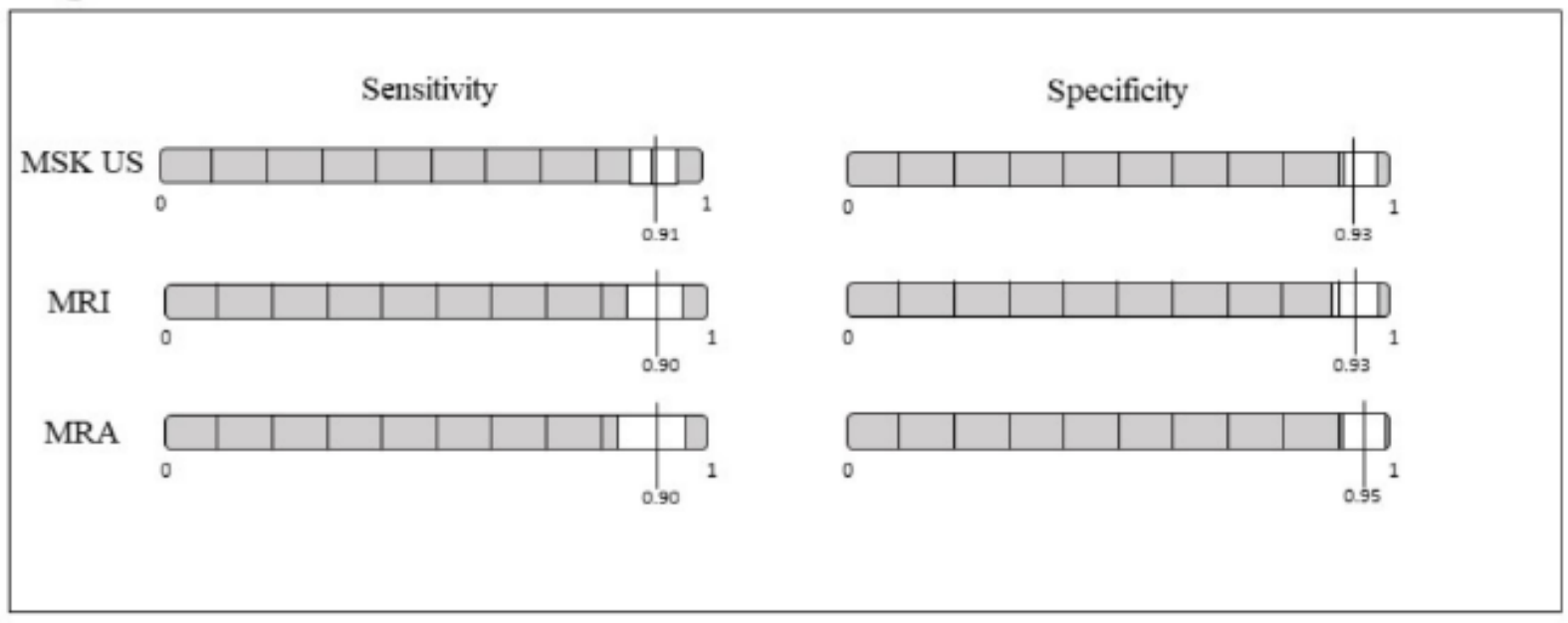

Figure 3 Partial thickness RC tears

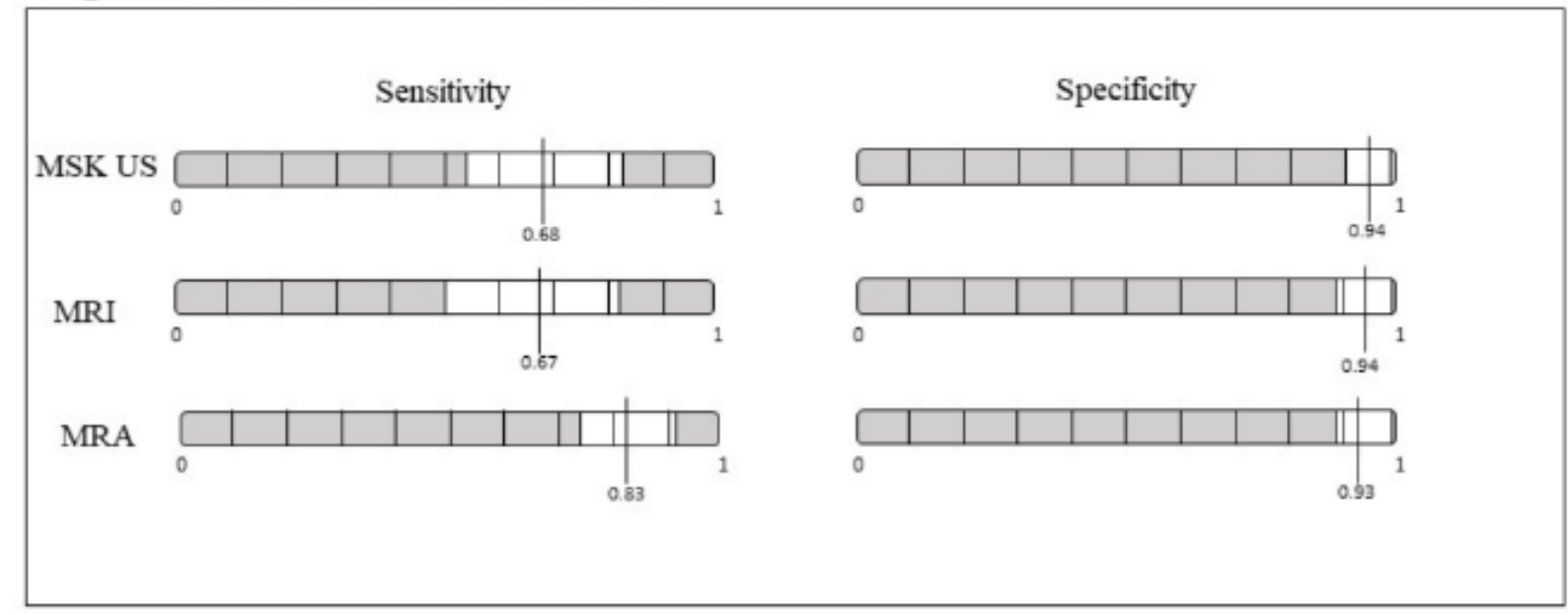

RC - Rotator Cuff 
though not statistically significant, data did reveal an advantage in image quality when using a $3.0 \mathrm{~T}$ MRI when compared to a $1.5 \mathrm{~T}$ machine. Finally, the sensitivity and specificity did not significantly vary between the results for full-thickness tears and partial-thickness rotator cuff tears for MRA. The literature did indicate that likelihood ratios supported the use of MRA in diagnosing partialthickness tears when compared to MSK US and MRI. 8

The limitation in this analysis was that the accuracy of MSK US as a diagnostic tool may be inflated. When the QUADAS-2 assessment was used, researchers believed there was bias that led to an increased likelihood of rotator cuff pathologies. This further resulted in an increased likelihood of findings through imaging. This review is also limited by the incomplete patient profiles reported resulting in incomplete data reporting. When exploring HSROC curves assessing diagnostic imaging techniques tools for their accuracy, it is evident that there is no significant difference between MSK US, MRI, and MRA. Each graph's curve was similar and had statistically significant numbers to help substantiate the accuracy of each tool. The only reason that MRI or MRA would be indicated over MSK US would be if there is an additional structure injured that may be contributing to the signs and symptoms the patient is presenting with. Additionally, insurance and instrument availability of diagnostic imagery may play a role in diagnostic image used though not considered by this review.

\section{CLINICAL BOTTOM LINE}

Patients who participate in sport and physical activity experience various pathologies including rotator cuff injuries. The sports medicine team is tasked with accurately advising their patients in the proper management of these shoulder injuries. For rotator cuff pathologies, it is imperative that full-thickness rotator cuff tears are diagnosed quickly and accurately as research indicates

Copyright $($ by Indiana State University All rights reserved. ISSN Online 2577-8188 surgical intervention yields the best outcome for patients and athlete to return to play. ${ }^{3}$ MSK US, MRI and MRA are the most commonly used diagnostic imaging tools for diagnosing rotator cuff pathology. The guiding manuscript indicates that there is no statistical difference between the ability of MSK US, MRI and MRA to diagnose rotator cuff pathology in individuals presenting with a possible rotator cuff pathology when performed and read by trained individuals. ${ }^{8}$ There is some evidence indicating that MRA has a slightly increased ability to detect partialthickness rotator cuff tears, though this finding is not considered to be statistically significant. ${ }^{8} \mathrm{MRI}$ and MRA are not cost or time effective due to their need for additional insurance authorization and appointments since imaging machines need to be reserved ahead of time. MSK US is recommended as an immediate screening tool, prior to ordering further imaging.

In the athletic population, there is an abundance of overuse shoulder pathology, especially in overhead sports such as baseball, softball, tennis and throwing events in track and field. ${ }^{10}$ Rotator cuff pathology can be managed through intensive therapeutic rehabilitation and modification of activities, though concern for worse injury is often present. Musculoskeletal ultrasound provides a cost effective, timely and reliable means of preliminarily evaluating a patient to assess the degree of injury and determine if surgery is warranted. Currently, MSK US can be performed by physician's trained in its uses and in the identification of musculoskeletal pathology or by an athletic trainer who has been trained and has the approval from their supervising physician. Further research is warranted to examine the cost benefit of an MSK US machine when portability and integration with various technological interfaces are considered. As the athletic training profession advances, all athletic trainers should be encouraged to take advantage of MSK training options. The use of MSK US in the athletic training facility can decrease the need for referral to an Volume 3 - Issue 3 - November 2020 
orthopedic physician as identification of a tendinopathy vs. a tear in the shoulder and other joint pathologies would be possible, ultimately decreasing cost and time for the patient. For the athletic trainer, MSK US machines are on average MSK US is recommended when rotator cuff pathology is suspected, but considerations should be made specific to the individual. Orthopedic evaluation should precede the use of MSK US as other pathologies may be present and not found upon evaluation with this diagnostic imaging technique.

\section{REFERENCES}

1. Balich SM, Sheley RC, Brown TR, Sauser DD, Quinn SF. MR imaging of the rotator cuff tendon: interobserver agreement and analysis of interpretive errors. Radiology. 1997; 204(1):191-194.

https://doi.org/10.1148/radiology.204.1.9 205245.

2. van der Windt DA, Koes BW, Boeke AJ, Deville W, De Jong BA, Bouter LM. Shoulder disorders in general practice: prognostic indicators of outcome. Br J Gen Pract. 1996; 46(410):519-523.

3. Lambers Heerspink FO, Dorrestijn $O$, van Raay JJ, Diercks RL. Specific patient-related prognostic factors for rotator cuff repair: a systematic review. I Shoulder Elbow Surg. 2014; 23(7):1073-1080. https://doi.org/10.1016/i.jse.2014.01.001.

4. Dinnes J, Loveman E, Mclntyre L, Waugh N. The effectiveness of diagnostic tests for the assessment of shoulder pain due to soft tissue disorders: a systematic review. Health Technol Assess. 2003; 7(29):iii, 1-166. https://doi.org/10.3310/hta7290.

5. Kanesa-Thasan RM, Nazarian LN, Parker L, Rao VM, Levin DC. Comparative Trends in Utilization of MRI and Ultrasound to Evaluate Nonspine Joint Disease 2003 to 2015. J Am Coll Radiol. 2018; 15(3 Pt A):402-407.

Copyright ( $)$ by Indiana State University All rights reserved. ISSN Online 2577-8188 https://doi.org/10.1016/i.jacr.2017.10.01

$\underline{5}$.

6. Leeflang MM. Systematic reviews and metaanalyses of diagnostic test accuracy. Clin Microbiol Infect. 2014; 20(2):105-113. https://doi.org/10.1111/1469-

0691.12474.

7. Harbord RM, Deeks JJ, Egger M, Whiting P, Sterne JA. A unification of models for metaanalysis of diagnostic accuracy studies. Biostatistics. 2007; 8(2):239-251. https://doi.org/10.1093/biostatistics/kxl00 4.

8. Roy JS, Braen C, Leblond J, et al. Diagnostic accuracy of ultrasonography, MRI and MR arthrography in the characterisation of rotator cuff disorders: a systematic review and meta-analysis. $\mathrm{Br} J$ Sports Med. 2015; 49(20):1316-1328.

https://doi.org/10.1136/bisports-2014094148.

9. Whiting PF, Rutjes AW, Westwood ME, et al. QUADAS-2: a revised tool for the quality assessment of diagnostic accuracy studies. Ann Intern Med. 2011; 155(8):529-536. https://doi.org/10.7326/0003-4819-1558-201110180-00009.

10. Zaremski JL, Wasser JG, Vincent HK. Mechanisms and Treatments for Shoulder Injuries in Overhead Throwing Athletes. Curr Sports Med Rep. 2017; 16(3):179-188. https://doi.org/10.1249/JSR.0000000000 $\underline{000361}$. 